\title{
Review
}

\section{Smokeless Tobacco Use in the United States Military: A Systematic Review}

\author{
Hannah E. Bergman, B.A., B.S., Yvonne M. Hunt, Ph.D., M.P.H., \& Erik Augustson, Ph.D., M.P.H. \\ Behavioral Research Program, Division of Cancer Control and Population Sciences, National Cancer Institute, Rockville, MD \\ Corresponding Author: Hannah E. Bergman, BA, BS, Case Western Reserve University, Department of Psychological Sciences \\ Psychology Program, 11220 Belleflower Road, Mather Memorial 109, Cleveland, OH 44106, USA. Telephone: 216-368-2686; \\ E-mail: hannah.bergman87@gmail.com
}

Received June 3, 2011; accepted August 19, 2011

\section{Abstract}

Introduction: Smokeless tobacco (ST) use represents an important target for intervention in the U.S. military population because it impairs "military readiness" and harms the health of the military. This paper aims to provide a systematic review of ST studies conducted in the U.S. military population in order to assess the content of existing ST research in this population, provide estimates of prevalence and clinically relevant use patterns, and discuss how these findings might be used to guide future ST research among this population.

Methods: We reviewed articles published through December 2010 using PubMed and PsycINFO databases, Google Scholar, and any relevant articles' reference lists. Inclusion criteria included focus on a U.S. military sample, English language, measured tobacco use, and ST prevalence was reported or could be calculated. To the extent possible, each article was coded for demographics, socioeconomic status, prevalence, amount, frequency, and length of use, and quit intentions/attempts.

Results: Thirty-nine articles met criteria for inclusion. Less than half focused primarily on ST use among military personnel. The remaining studies measured ST use in the context of other behaviors. Findings related to clinically relevant behaviors included a need for more cohort and intervention studies, a better understanding of ST use in combination with cigarettes (i.e., concurrent use), and identifying risk factors for ST initiation and use.

Discussion: ST use is prevalent among military personnel, as is concurrent use of cigarettes and ST. We provide a number of recommendations to guide future research in this important, yet understudied, area.

\section{Introduction}

Approximately 1.5 million Americans are active duty members of the U.S. Armed Forces, serving within one of its five branches: Air Force, Army, Coast Guard, Marine Corps, and Navy (U.S. Department of Defense [DoD], 2011). Despite notable progress in reducing the prevalence of cigarette smoking in the military,

doi: $10.1093 / \mathrm{ntr} / \mathrm{ntr} 216$

Advance Access published on November 28, 2011

Published by Oxford University Press on behalf of the Society for Research on Nicotine and Tobacco 2011. smokeless tobacco (ST) use is increasing (Institute of Medicine [IOM], 2009). Furthermore, many military personnel use cigarettes and ST concurrently, compounding the health hazards of using either product alone and maintaining the addiction to nicotine (IOM, 2009). According to a recent survey of active duty personnel, nearly half $(41.2 \%)$ reported using one or more forms of tobacco in the past month (Rae Olmsted, Bray, Guzman, Williams, \& Kruger, 2011).

Significant differences in ST use rates exist between the military and civilian populations. In 2008, the overall prevalence of ST use among active duty personnel was $14 \%$ (Bray et al., 2009). In comparison, $3.5 \%$ of civilians ( $\geq 18$ years) used ST (Substance Abuse and Mental Health Services Administration [SAMHSA], 2010). In both populations, ST use is highest among young adult (aged 18-25) White males. However, even in this demographic group, those in the military use ST at almost twice the rate of their civilian counterparts (19\% vs. 11.4\%; Bray et al., 2009; SAMHSA, 2010). Significant differences also exist in rates of ST use between the individual service branches. The Marine Corps has the highest prevalence (22\%), followed by the Army (16\%), Coast Guard (13\%), Navy (10\%), and Air Force (9\%; Bray et al., 2009).

ST use is associated with numerous adverse health outcomes, including cancers of the mouth, throat, and pancreas (Boffetta, Hecht, Gray, Gupta, \& Straif, 2008; International Agency for Research on Cancer, 2007; Warnakulasuriya, 2009); gum disease (Chu, Tatakis, \& Wee, 2010; Warnakulasuriya et al., 2010); oral lesions (Warnakulasuriya et al., 2010); and cardiovascular disease (Piano et al., 2010; Yatsuya \& Folsom, 2010). Additional health risks are incurred with concurrent use of cigarettes and ST (IOM, 2009). Moreover, there are a variety of tobacco-associated negative outcomes relevant to the military population. For example, impaired night vision and delayed healing from injures and wounds can be particularly detrimental to "military readiness" (Bray et al., 2006), especially to those on combat deployment.

A number of influential reports have been issued on the problem of tobacco use in the military, though none have focused specifically on the problem of ST use. A recent review concluded that ST use in the military is a growing problem and 
should be targeted by tobacco cessation treatment efforts (IOM, 2009). The DoD also supports research on tobacco use and cessation in military personnel and has been tracking ST use since 1985 (Bray et al., 2009). This data have proved extremely useful with regard to describing trends in ST use over time.

The current review aims to extend the knowledge gained from these reports by undertaking a systematic review of ST studies conducted in the U.S. military population. The goals of the review are to (a) assess the content of existing ST research in this population, (b) provide estimates of prevalence, (c) identify patterns of potential clinical importance associated with ST use, and (d) discuss how these findings might be used to inform needed ST research among military personnel. Identifying and better understanding predictors of ST use initiation, the impact of concurrent use of cigarettes and ST, and what prevention and cessation interventions are effective and efficacious will positively impact the health of the military.

\section{Methods}

\section{Identification of Studies}

Articles were identified through a literature search using PubMed and PsycINFO databases, Google Scholar, and any relevant articles' reference lists. The phrase "smokeless tobacco military" was used in each search as well as several variations on the keyword "smokeless tobacco" (i.e., "dip," "chew," "snuff," and "Snus").

\section{Inclusion Criteria}

A study was included if it was (a) published in December 2010 or before, (b) specifically about the U.S. military, (c) written in English, (d) tobacco use was defined (i.e., smokeless, chew, dip, snuff), and (e) the ST prevalence was reported or could be calculated. If more than one population sample was included in the study (e.g., Chisick, Poindexter, \& York, 1998; Fitzpatrick \& Shannon, 1992), each sample was coded separately to ensure that all sample characteristics were captured and to best represent the diversity of ST use in this review.

Numerous variables related to the ST user population were coded for (e.g., ST use level, demographics, frequency of ST use, etc.). Supplementary Table 1 lists the ST user population variables reported by each article. We only reported percentages that were either provided or that we were able to calculate (i.e., excluded variables if they were reported as "most" or "majority") for variables associated with the ST user population.

The categorization of ST use level prevalence (i.e., current, daily/regular) was based on the terminology and definitions provided in each of the 39 articles. If the ST use level was not specified, we determined the category for the reported ST use prevalence. For studies that reported concurrent use, we categorized these prevalence rates into one of three categories: (a) ST users who also smoke cigarettes, (b) cigarette smokers who also use ST, and (c) users of both cigarettes and ST (primary product not identified; See Supplementary Table 1 for more information on ST/concurrent use definitions and prevalence rates).

When providing summary data for a group of studies (e.g., education level of ST users), we reported the range of percentages. We also reported the calculated weighted mean prevalence rates for all ST use levels and concurrent use, along with the prevalence ranges, to account for the varying sample sizes. The weighted mean prevalence rates were calculated by multiplying the sample sizes by prevalence rates and dividing by the total sample size. Three samples were excluded from this calculation because $100 \%$ of participants were ST users. For the cohort and intervention studies, only baseline data were used when calculating the range of percentages and weighted mean prevalence rates.

\section{Results}

\section{Description of Studies}

The initial literature search identified 45 articles; 39 articles met criteria for review (6 did not meet inclusion criteria). Supplementary Table 2 provides a summary of the 39 articles, including military branch(es) and sample description (i.e., sample size, duty status, sampling method, etc.). Of these, 29 were cross-sectional studies, 5 were intervention studies, 4 were cohort studies, and 1 was a review. The majority of the studies $(n=24,61.54 \%)$ were published between 2000 and 2010, suggesting that the body of literature is still relatively nascent. The sample size ranged from 38 (Sridhar et al., 2003) to 33,215 (Klesges et al., 2006).

Thirteen articles were exclusively about ST use (i.e., ST user only sample, ST cessation intervention). The remainder either looked at ST use within the context of general tobacco use (i.e., concurrent use, interventions that target tobacco use; $n=18$ ) or ST use within the context of other health behaviors/concerns (i.e., tobacco use and military training exercises, alcohol, tobacco, and other drug use; $n=8$ ).

The majority of studies were conducted with Air Force personnel only $(n=16)$, followed by Army only samples $(n=10)$, Navy only samples $(n=3)$, and Marine Corps only samples $(n=2)$. The remainder $(n=8)$ were conducted with samples drawn from multiple branches. Eighteen studies were conducted with active duty personnel only, 17 studies with basic military trainees only, 1 study with recruits (data were collected at the inprocessing center 3 days before the start of basic military training [BMT]; Chisick et al., 1998), and 1 study was conducted at the U.S. Military Academy at West Point (Knapik, Reynolds, \& Barson, 1999). Two studies had participants from various duty statuses (Chisick et al., 1998; Kenny, Quigley, \& Regennitter, 1996). One study did not report the sample's duty status (Shipley, Tresch, Tracey, \& Wilcox, 2002).

\section{General Characteristics of the ST User Population}

Across the 39 studies, the majority of ST users were enlisted White males under the age of 30. One study looked at ST use within a female only sample (Vander Weg, DeBon, et al., 2005).

The ST prevalence rate was reported in a number of ways including current, daily/regular, occasional, experimental, lifetime, and/or former use. Supplementary Table 1 highlights that there was no uniform definition for each of the ST use levels. These differences and their implications are addressed in the "Future Directions" section. Current use was reported in 23 
studies. The range of prevalence was .4\%-50\%, and the calculated weighted mean prevalence of current use was $9.4 \%$ in 21 studies. Participants in two studies were all current users (Peterson et al., 2007; Severson et al., 2009); they were excluded from the range and weighted mean prevalence calculations. Daily/regular use was reported in 12 studies. The range of prevalence was $2.0 \%-71.5 \%$, and the calculated weighted mean prevalence was $7.2 \%$ in 11 studies. Participants in one study were all daily/ regular users (Cigrang, Severson, \& Peterson, 2002); they were excluded from the range and weighted mean prevalence calculations. Occasional use was reported in five studies; the range of prevalence was $1.2 \%-8.8 \%$. The calculated weighted mean prevalence was $4 \%$. Experimental use was reported in eight studies; the range of prevalence was $6.6 \%-24.7 \%$. The calculated weighted mean prevalence was $14.4 \%$. Lifetime use was reported in four studies; the range of prevalence was $7.9 \%-43.1 \%$. The calculated weighted mean prevalence was $24.9 \%$. Former use was reported in nine studies; the range of prevalence was $0.2 \%-$ $30.6 \%$. The calculated weighted mean prevalence was $3.6 \%$.

Concurrent use of cigarettes and ST was reported in 19 studies and was categorized in one of three ways: (a) ST users who also smoke cigarettes, (b) cigarette smokers who also use ST, or (c) individuals who use both (primary tobacco product not identified). Concurrent use, defined as ST users who also smoke cigarettes, was reported in seven studies; the range of prevalence was $7.0 \%-64.4 \%$. The calculated weighted mean prevalence was $47.3 \%$. Concurrent use, defined as cigarette smokers who also use ST, was reported in five studies; the range of prevalence was $4.5 \%-41.8 \%$. The calculated weighted mean prevalence was $18.3 \%$. Concurrent use, defined as individuals who use both tobacco products, was reported in six studies; the range of prevalence was $4.8 \%-68.6 \%$. The calculated weighted mean prevalence was $39.4 \%$. Of these 19 studies, 9 reported the gender of concurrent users; the majority were male.

One study compared ST use of daily light cigarette smokers versus intermittent cigarette smokers (Cooper et al., 2010). Relative to daily light smokers, intermittent smokers were more likely to use ST daily and occasionally ( $7 \%$ vs. $2 \% ; 7 \%$ vs. $5 \%$ ). Relative to intermittent smokers, daily light smokers used ST more experimentally ( $24 \%$ vs. $22 \%$ ). Klesges et al. (2006) reported cigarette smokers' daily, experimental, and former ST use for the smoking intervention group. In this group, $14.3 \%$ were daily ST users, $24.7 \%$ were experimental users, and 5.7\% were former users.

\section{Specific Characteristics of the ST User Population \\ Other Demographics}

Four studies reported the marital status of ST users, with mixed findings. Two studies found that the majority of users were married (Peterson et al., 2007; Severson et al., 2009), while the majority of users in the other two studies were single (Ebbert et al., 2006; Haddock et al., 2001).

One study reported the hometown population size of snuff (i.e., ground tobacco leaves) and chew (i.e., crushed tobacco leaves) users (Daly \& Pierson, 1990). More snuff users (41.7\%) came from a hometown of less than 25,000 people than from any other population size category. Most chew users (52.1\%) came from a hometown of less than 25,000 people. Two studies
(Daly \& Pierson, 1990; Vander Weg, DeBon, et al., 2005) reported the geographic origin of region of ST users: $11.0 \%-$ $13.6 \%$ were from the Northeast, $22.9 \%-26.0 \%$ were from the South/Southeast, 7\%-13\% were from the Southwest, $29.0 \%-$ $35.6 \%$ were from the Midwest, and 21\%-28\% were from the West/Pacific.

\section{Socioeconomic Status}

Eight studies reported the education level of ST users. Two studies reported that the majority of users had 12 years or less of education (Lando, Haddock, Klesges, Talcott, \& Jensen, 1999; Vander Weg et al., 2008). Five studies reported that $22.1 \%-78.7 \%$ of users had some college education. Two studies reported that $21.8 \%$ (Kenny et al., 1996) and 26.9\% (Severson et al., 2009) of users either completed a 4 -year college degree and/or continued with graduate school. All participants in one study were U.S. Army general medical officers, who were surveyed about their knowledge on smoking cessation, training, practice, and to report their own tobacco use (7\% reported regular ST use; Hepburn, Johnson, Ward, \& Longfield, 2000).

A majority of ST users in two studies reported an income level of more than $\$ 20,000$ (Haddock et al., 2001; Lando et al., 1999). One study reported the different income levels of ST users: $21.1 \%$ reported an income of less than $\$ 25,000,23.3 \%$ reported $\$ 25,000-\$ 45,000,26.1 \%$ reported $\$ 45,000-\$ 70,000$, and $29.6 \%$ reported more than $\$ 70,000$ (Vander Weg et al., 2008).

\section{Amount of ST Used}

Ten studies reported the amount of ST used, with mixed findings. Four studies reported that a majority of users used one can/tin or less per day. One study reported that $51 \%$ of ST users used more than one can/tin/pouch per day (Burns \& Williams, 1995). Three studies reported that the majority of users used less than two cans/tins/pouches per week. One study reported that $64.3 \%$ of ST users used less than two cans/tins/pouches per week (Williams, Hermesch, Gackstetter, Lando, \& Fiedler, 1996). One study reported that the average number of cans used per day was 0.8 (Sridhar et al., 2003).

\section{Frequency of ST Use}

Six studies reported the frequency of ST use. Two studies reported average minutes of daily use (Daly \& Pierson, 1990; Kenny et al., 1996). Daly and Pierson found that snuff users averaged more minutes of use per day compared with chew users (104.1 vs. $72.1 \mathrm{~min}$ ). Kenny et al. reported that users used for an average of $25.8 \mathrm{~min}$ per day. Two studies reported the average number of days of ST use and the percentage of those using ST within 30 min of waking (Peterson et al., 2007; Severson et al., 2009). Both studies found that the average number of days per week of use was 6.2 days and that $23.9 \%$ used within $30 \mathrm{~min}$ of waking. Martin, Brown, Eifler, and Houston (1999) found that 4 days was average number of days since last use. One study reported that ST users averaged seven dips per day (Trent, Hilton, \& Melcer, 2007).

\section{Length of ST Use}

Six studies reported the average number of years of ST use (range $=2.98-12.80$ years).

\section{Quitting Intentions/Tobacco Outcomes}

Four studies reported quitting intentions of ST users. Peterson et al. (2007) and Severson et al. (2009) reported the percentage 
of users who made a quit attempt within the past year and the average score on the readiness to quit scale $(0=$ no thought of quitting to 10 = taking action to quit; see Biener \& Abrams, 1991). Peterson et al. reported that $45.9 \%$ of users had made a quit attempt in the last year and averaged a 6.4 on the readiness to quit scale; Severson et al. reported that $45.4 \%$ of users had made a quit attempt in the last year and averaged a 6.4 on the scale. Grasser and Childers (1997), and McClellan, Olde, Freeman, Mann, and Rotruck (2010) reported that it took more than three quit attempts to be successful.

\section{Cohort Studies}

There were four cohort studies, two studies examined the predictors of tobacco use (Ebbert et al., 2006; Haddock et al., 2001) and two studies examined the rate of tobacco use before and after BMT and the impact of the mandatory tobacco ban during BMT (Klesges, Sherrill-Mittleman, Ebbert, Talcott, \& DeBon, 2010; Williams et al., 1996). The participants in all four studies were Air Force trainees. All trainees during BMT are tobacco free because of a mandatory ban of tobacco products during this time. This ban includes all tobacco products (i.e., ST, cigarettes, cigars, etc.) as well as tobacco cessation aids (i.e., nicotine gum, patch, medication, etc.; Klesges et al., 2006). Therefore, the ST use level reported at baseline represents the history of ST use prior to entering BMT.

Ebbert et al. (2006) examined the predictors of ST use at 1-year follow-up. They found that those who were experimental ST users at baseline (second week of BMT) were most likely to report any, current, or daily ST use at 1-year follow-up compared with those who were classified as never ST users at baseline (19.3\%, $8.3 \%, 6.5 \%$ vs. $3.9 \%, 1.5 \%, 1.2 \%$, respectively). Current cigarette smoking at baseline was also a significant predictor of ST use at 1-year follow-up. Individuals whose ST status changed from never user to ST user were more likely to be male than female. Ebbert et al. concluded that males who were current smokers at baseline were at the greatest risk to be ST users at follow-up.

Haddock et al. (2001) examined the role of ST use as a predictor of future cigarette use. They found that the individuals most likely to be cigarette smokers at 1-year follow-up were those who were current and former ST users at baseline (upon entrance to BMT) as compared to baseline never ST users $(27.0 \%, 26.3 \%$ vs. $12.9 \%)$.

Klesges et al. (2010) found that of the 3.7\% participants who reported using ST only at baseline (second week of BMT), $32.6 \%$ continued their ST use, $14.0 \%$ had switched to cigarette smoking only, $14.5 \%$ were concurrent users, and $38.9 \%$ had quit ST use at 1-year follow-up. Among the $33.5 \%$ of participants who reported cigarette smoking only at baseline, $69.8 \%$ continued to smoke cigarettes, $.9 \%$ had switched to ST use only, and $5.6 \%$ were concurrent users at 1-year follow-up. Among the $4.8 \%$ of participants who reported concurrent use of cigarettes and ST at baseline, $25.3 \%$ continued to be concurrent users, $12.0 \%$ had switched to ST use only, and $42.2 \%$ had switched to cigarette smoking only. At 1-year follow-up, most cigarette and ST users had either maintained or increased their tobacco use.

Williams et al. (1996) found that the overall ST prevalence for males decreased between baseline (second day of BMT) and 90 days after BMT (12.7\% to 9.8\%). The study also looked at the prevalence rate of male ST users before and after BMT based on the amount of ST used. The authors reported that males who used less than two cans per week were more likely to start using again after BMT than those who used more than two cans per week before BMT ( $<2$ cans: $8.1 \%-6.2 \%$ [not significant]; $>2$ cans: $4.6 \%-3.6 \%$ [not significant]). The authors reported that concurrent use increased from before to after BMT, 13.0\%$16.4 \%$ (not significant). The study concluded that the mandatory tobacco abstinence during BMT had a positive, though small, effect on ST use after BMT.

\section{Intervention Studies}

Five studies assessed the efficacy of ST use cessation interventions (Cigrang et al., 2002; Klesges et al., 2006; Morgan, 2001; Severson et al., 2009; Shipley et al., 2002). Positive treatment outcomes were seen across all five studies.

Cigrang et al. (2002) wanted to determine if a minimalcontact behavioral intervention (i.e., brief telephone counseling sessions) that integrated motivational interviewing techniques was efficacious and effective in a sample of Air Force active duty personnel. The participants were randomly assigned to the intervention or usual-care condition. The study found that the intervention effect was significant (compared with the usualcare group) at 3-month follow-up (41\% vs. $17 \%$ ) but not at 6-month follow-up (37\% vs. 19\%) for ST quit rates.

Severson et al. (2009) wanted to see if the results found in the Cigrang et al. (2002) study would extend to a sample of active duty personnel from multiple branches. The participants were randomly assigned to the intervention or usual-care condition. Severson et al. reported that the intervention (compared with the usual-care group) was significant for prolonged ST abstinence at 3 -month (26.0\% vs. $10.7 \%)$ and 6-month $(16.8 \%$ vs. $6.4 \%$ ) follow-ups.

Klesges et al. (2006) randomly assigned Air Force trainees to the tobacco control intervention or control condition. For participants in the intervention condition, they were designated to one of three "behavioral treatment modules" based on their history of cigarette and/or ST use at baseline (tobacco history based on use before entering BMT). The study used retired BMT military training instructors to lead the intervention sessions. The study found that participants in the ST intervention group were significantly more likely to report continuous abstinence (compared with the control group) at 1-year follow-up (33.7\% vs. $28.3 \%)$.

Morgan (2001) made a presentation about tobacco's negative health effects and had the unit commander reinforce those points to a convenience sample of Army active duty personnel who were tobacco users (cigarettes and/or ST). Morgan reported that $61 \%$ ST users were more likely to reduce their use (15\%) or quit (46\%) as compared with users of cigarettes only and users of both cigarettes and ST at 1-month follow-up.

Shipley et al. (2002) used a behavioral and pharmacological component with an Air Force sample. The behavioral intervention was done in individual sessions. For the pharmacological intervention, ST users were offered either the nicotine patch and bupropion, nicotine gum and bupropion, nicotine patch alone, or bupropion alone. The study reported that $78 \%$ of ST users reported abstinence at 6-month follow-up. 


\section{Discussion}

Overall, the majority of the 39 studies have been cross-sectional and conducted with Air Force active duty personnel. Notably, although many of the demographics match what we might expect in a representative military sample, most studies were not designed to be representative of the military. The majority of the studies examined ST use in the context of broader tobacco use patterns $(n=18)$. In addition, there were only a small number of cohort and intervention studies.

Only four cohort studies were identified for this review, all of which were conducted with Air Force trainees. Three of these studies examined successive recruit classes over the course of 1 year, providing numerous populations and data collection timepoints (Ebbert et al., 2006; Haddock et al., 2001; Klesges et al., 2010). All four cohort studies found that both ST use and cigarette smoking are predictors of use of the other tobacco product and that most ST users continued their use 1 year post-BMT.

The current review also highlighted a number of variables that were not always reported for the ST user population: amount ( $n=10$ studies), education level $(n=8)$, frequency of use $(n=6)$, length of use $(n=6)$, quit intentions/tobacco outcomes $(n=5)$, marital status $(n=4)$, income level $(n=3)$, geographic region of origin $(n=2)$, and hometown population size $(n=1)$. More consistent reporting of these variables would improve our understanding of ST use in the military.

The current review found wide variability in the reported ST use level and concurrent use prevalence rates. We suspect that this variability is a result of the different sampling methods and sample sizes used, and how each of the ST use levels and concurrent use was defined. Many of the studies oversampled a particular group (e.g., ST users or females) or used a convenience sample, thus contributing to the wide range of ST use rates reported. For example, a high ST use rate was reported in a majority ST user sample (Bahrke, Poland, Baur, \& Connors, 1988) and low ST use rate was reported in a female only sample (Vander Weg, DeBon, et al., 2005).

Varying sample sizes (ranged $=38-33,215)$ also influenced the ST use level and concurrent use prevalence rates reported. The widest variation in reported ST use, in relation to sample size, was for current, daily/regular, experimental, and former use and also across the all three concurrent use categories. In most cases, ST use prevalence was highest among small samples (e.g., 50\% current ST users among 38 participants; Sridhar et al., 2003) and lowest among large samples (e.g., 4.1\% current ST users among 32,144 participants; Ward et al., 2003).

Finally, the methods used to define the different ST use levels could account for the wide variability of prevalence rates reported. Klesges et al. (2011) examined how different definitions of concurrent use affected reported prevalence rates. They found that the concurrent use rate reported depended greatly on the definition of concurrent use (e.g., both cigarettes and ST were used daily or one product was used daily and the other used nondaily). How the ST use levels were operationalized potentially influenced the prevalence rates being reported in the current review (as seen in Supplementary Table 1). Although we grouped the ST use levels based on how each study measured ST use, we could not necessarily account for the different ways that a particular ST use level was being defined. For example, some definitions of current ST use included ST use in the prior thirty days (Ames, Cunradi, \& Moore, 2002), using ST at least once per day (Haddock et al., 2001), and using ST regularly or occasionally (Vander Weg et al., 2008).

As for concurrent use, since most of the studies did not define concurrent use (i.e., identifying the primary and secondary product based on frequency of use), we used our best judgment in determining how concurrent prevalence rates were being reported (i.e., ST users who also smoke cigarettes, etc.). It is possible (and unknowingly) that we potentially categorized the reported concurrent use into the wrong category. However, we do not feel that this confounds our results on concurrent use.

Although the current findings highlight the high overall ST prevalence, we were not able to systematically compare prevalence rates between branches due to lack of sufficient data from the current published studies. Branch comparisons are important to help us identify similarities and differences of ST use and users in the different branches. Such comparisons are available elsewhere (e.g., Bray et al., 2009); however, the composition of the studies included in this review did not allow for meaningful comparisons between service branches or comparison of other factors related to ST use between the branches. Of the 39 studies, 16 studies were conducted with Air Force personnel, while only 2 studies were conducted with Marine Corps samples. This is important because other data indicate that the Air Force has the lowest prevalence of ST use (9\%), while the Marine Corps has the highest prevalence of ST use (22\%; Bray et al., 2009). The observed imbalance of ST use studies among the service branches may be attributable, at least in part, to varying institutional review board (IRB) requirements across the service branches. Because each branch has its own set of procedures in place for obtaining IRB and other military approvals, the obstacles to engaging in data collection across the service branches can be formidable. A standardized or centralized IRB approval process would help to reduce this limiting factor.

\section{Clinical Implications}

The results of this literature review have a number of important clinical implications. Broadly, the findings indicate that ST use represents an important target for intervention in the U.S. military population because it impairs "military readiness" and hurts the health of the military. Prevalence estimates of ST use across all studies were high, even when compared with demographically similar nonmilitary populations, suggesting that military personnel carry a disproportionate burden of ST use and its associated risks. Targeted interventions are needed to reduce ST use in this population.

In addition to a high overall prevalence of ST use, the review found that many military personnel use both cigarettes and ST concurrently. Concurrent use was reported in nearly half of the studies $(n=19)$. From an intervention perspective, concurrent use presents a unique challenge for intervening with ST use in population. Dual users are exposed to higher levels of nicotine (Wetter et al., 2002), may be less likely to make a quit attempt (Hatsukami \& Severson, 1999; Tomar, Alpert, \& Connolly, 2010; Walsh et al., 2010), and more likely to relapse (Wetter et al., 2002). The extent to which existing cessation approaches are effective in addressing concurrent use patterns 
of tobacco use is largely unknown. New strategies may be needed to effectively address the problem of concurrent use in this population.

It is noteworthy that the current review identified only a handful of ST intervention studies involving military personnel $(n=5)$. Three of these studies were conducted in the Air Force, one in the Army, and one with participants from multiple branches. Only one intervention study had both a behavioral and a pharmacological component (Shipley et al., 2002). The results of these studies suggest that it is possible to effectively intervene with ST use in this population. However, more intervention studies are clearly needed.

\section{Future Directions}

To address significant gaps in research on ST use in the military, we offer the following recommendations.

First, more longitudinal and cohort studies of ST use are needed in this population. Such studies would provide a better understanding of important critical periods for intervening with ST use. In particular, more research is needed that examines the developmental trajectories of tobacco use among military personnel. Critical timepoints for studying ST use would include before BMT, after BMT/tech school, transition between training and first duty station, and deployment. Other factors such as type of deployment (e.g., inside or outside the wire), type of duty station (e.g., bases with restricted or liberal tobacco policies), and service branch also represent important variables to include in these studies.

Furthermore, there is a need for greater representation of the entire military (i.e., all five branches) in cohort studies. Although the four cohort studies identified in this review provided valuable information about ST use before and after BMT, all of them were conducted with Air Force samples, and thus, the results cannot necessarily be generalized to other branches. A cohort study conducted across all branches of the military would further elucidate military-wide patterns of ST use as well as similarities and differences between the different branches and duty statuses.

Second, there is a critical need for more intervention studies in this population. ST prevalence rates among military personnel are disproportionately high compared with the civilian population. Yet, this review identified only a handful of intervention trials that have been conducted to reduce ST use among service members. Of the five intervention studies examined in this review, three were conducted with Air Force personnel. One research question is whether the Air Force ST interventions would produce similar ST cessation rates in other branches.

Successfully intervening with ST use in this population will require a better understanding of the clinical factors that are unique to military personnel. Specifically, the development of tailored approaches will be important for addressing patterns of concurrent use and intervening with ST use during BMT and deployment.

Third, studies should address other at-risk populations for ST use in the military, such as deployed personnel. To have the largest impact, cohort and intervention research should focus on subgroups of military personnel with high ST use prevalence:
White males, ages 18-25 years, members of Army or Marine Corps service branches, and deployed personnel (especially those deployed to combat zones). In addition, targeted prevention approaches are needed to identify and intervene with those most at risk for initiating ST use upon entering the military.

Our review identified only one study that looked at ST use among deployed personnel during Operation Desert Storm (Forgas, Meyer, \& Cohen, 1996); however, from an intervention perspective, deployed personnel represent a high priority population. According to a recent IOM report, the use of ST is increasing in military populations, particularly among service members deployed to Iraq and Afghanistan (IOM, 2009). Deployed personnel face unique challenges that may make intervening with tobacco use particularly difficult. Personnel deployed to high-risk theaters of operation commonly face challenges such as unreliable or nonexistent Internet access and/ or cell phone service, inadequate services, poor living conditions, as well as the daily stress (physically and/or mentally) of the job. More research is needed in this area to inform intervention approaches targeting deployed personnel.

Fourth, additional research focused on concurrent use is needed. Another clinically relevant finding from the review is that many military personnel use cigarettes and ST concurrently. More research is needed to understand patterns of concurrent use in military personnel and identify strategies to effectively address the problem. As part of a broader initiative to stimulate research on ST use, the National Cancer Institute (NCI) recently funded a large cohort study designed to identify predictors of ST and concurrent use in approximately 30,000 Air Force trainees. The results of this study will inform the limited literature on the use of multiple tobacco products in the U.S. military (U.S. Department of Health and Human Services, National Institutes of Health, NCI, 2010).

The recent emergence of alternative tobacco products and how these products are marketed to military personnel represent a key area for future study. For example, the tobacco companies have been aggressively marketing new ST products, such as spitless tobacco (e.g., Snus) and dissolvables (e.g., lozenges), and the military population has historically been a targeted population for tobacco products (Joseph, Muggli, Pearson, \& Lando, 2005). Haddock et al. (2008) found that in an examination of tobacco advertisements found in the 2005 issues of Military Times magazine, the only tobacco advertisements were for ST products, and these products were advertised repeatedly. At the same time, there were relatively few articles about tobacco control in the magazine.

Fifth, further research needs to be done on the perception of ST use in the military. Previous research suggests, even though the military has many tobacco control policies in effect, that there continues to be a pro-tobacco culture within some segments of the military (Conway, 1998; Haddock et al., 2009). If this is the case, this type of institutionalized culture could undermine efforts to effectively change usage patterns. A better understanding of factors such as differences in the individual branch tobacco policies, attitudes toward tobacco, and the branch/military culture and how they relate to the military's attitude toward tobacco could identify obstacles that need to be addressed in order to facilitate efforts to reduce tobacco use and the related health burden within military personnel. 
Finally, standardized operational definitions of the different ST use levels are needed. This recommendation goes beyond ST research in the military and underscores the importance of having standardized definitions in the tobacco control field (Klesges et al., 2011).

Although a majority of the studies used the terms current, daily/regular, occasional, experimental, lifetime, and/or former to describe the ST users, there was not always a consistency in how each of these terms were defined (as seen in Supplementary Table 1). We believe that a common definition for each of the ST use levels would cut down on the variability observed in the prevalence rates reported in the current review. Moreover, universal terminology and definitions would provide ST researchers a common ground in how to report their findings. This would make comparisons between results easier to interpret since the prevalence rates reported would be based on the same terminology and definition.

\section{Conclusions}

With a combined strength of over 1.5 million active duty personnel (U.S. DoD, 2011) and more than 1.5 million Reserve/ National Guard personnel (U.S. Census Bureau, 2011), the U.S. military makes the DoD the largest federal employer. Yet, despite this vast number, they remain an underrepresented population with respect to tobacco control. The military has an alarmingly high ST prevalence compared with the rest of the U.S. population. Yet, there has been a lack of research on ST use in the military, which has resulted in a limited knowledge base regarding factors related to ST use. The results of this review underscore the need for better surveillance research and more intervention studies of ST use in the U.S. military population.

\section{Supplementary Material}

Supplementary Tables 1 and 2 can be found online at http://www. ntr.oxfordjournals.org

\section{Funding}

The corresponding author was funded as a Cancer Research Training Award Fellow at the NCI, National Institutes of Health, from September 2009 to August 2011.

\section{Declaration of Interests}

None declared.

\section{Acknowledgments}

The authors would like to thank Michele Bloch, M.D., Ph.D., Mark Parascandola, Ph.D., M.P.H., Sarah Evans, Ph.D., and Gary Roberson for their contributions in preparation of this manuscript. The authors would also like to thank the two anonymous reviewers for their helpful suggestions.

\section{References}

Ames, G. M., Cunradi, C. B., \& Moore, R. S. (2002). Alcohol, tobacco, and drug use among young adults prior to entering the military. Prevention Science, 3, 135-144. doi:10.1023/A:10154 35401380

Bahrke, M. S., Baur, T. S., Poland, D. F., \& Connors, D. F. (1988). Tobacco use and performance on the U.S. Army physical fitness test. Military Medicine, 153, 229-235. Retrieved from http://psycnet.apa.org

Biener, L., \& Abrams, D. B. (1991). The contemplation ladder: Validation of a measure of readiness to consider smoking cessation. Health Psychology, 10, 360-365. doi:10.1037/0278-6133.10. 5.360

Bray, R. M., Hourani, L. L., Olmsted, M. L. R., Witt, M., Brown, J. M., Pemberton, M. R., et al. (2006). 2005 Department of Defense survey of health related behaviors among active duty military personnel: A component of the Defense Lifestyle Assessment Program (DLAP). Retrieved from http://www.health.mil/

Bray, R. M., Pemberton, M. R., Hourani, L. L., Witt, M., Olmsted, K. L. R., Brown, J. M., et al. (2009). 2008 Department of Defense survey of health related behaviors among active duty military personnel: A component of the Defense Lifestyle Assessment Program (DLAP). Retrieved from https://dlap.rti.org/active/ Home.aspx

Boffetta, P., Hecht, S., Gray, N., Gupta, P., \& Straif, K. (2008). Smokeless tobacco and cancer. The Lancet, 9, 667-675. doi:10.1016/S1470-2045(08)70173-6

Burns, J. C., \& Williams, L. N., Jr. (1995). A survey to determine the knowledge of military members about the hazards of tobacco use, and a resulting tobacco-hazard education project. Journal of Cancer Education, 10, 37-40. doi:10.1080/08858199509 528326

Chisick, M. C., Poindexter, F. R., \& York, A. K. (1998). Comparing tobacco use among incoming recruits and military personnel on active duty in the United States. Tobacco Control, 7, 236-240. doi:10.1136/tc.7.3.236

Chu, Y. H., Tatakis, D. N., \& Wee, A. G. (2010). Smokeless tobacco use and periodontal health in a rural male population. Journal of Periodontology, 81, 848-854. doi:10.1902/jop.2010. 090310

Cigrang, J. A., Severson, H. H., \& Peterson, A. L. (2002). Pilot evaluation of a population-based health intervention for reducing use of smokeless tobacco. Nicotine \& Tobacco Research, 4, 127-131. doi:10.1080/14622200110101603

Conway, T. L. (1998). Tobacco use and the United States military: A longstanding problem. Tobacco Control, 7, 219-221. doi:10.1136/tc.7.3.219

Cooper, T. V., Taylor, T., Murray, A., DeBon, M. W., Vander Weg, M., Klesges, R. C., et al. (2010). Differences between intermittent and light daily smokers in a population of U.S. military recruits. Nicotine \& Tobacco Research, 12, 465-473. doi:10.1093/ntr/ntq025 
Daly, M. B., \& Pierson, W. (1990). The use of smokeless tobacco among basic airmen. American Journal of Health Promotion, 4, 266-269. Retrieved from http://psycnet.apa.org

Ebbert, J. O., Haddock, C. K., Vander, W. M., Klesges, R. C., Poston, W. S., \& DeBon, M. (2006). Predictors of smokeless tobacco initiation in a young adult military cohort. American Journal of Health Behavior, 30, 103-112. doi:10.5555/ajhb. 2006.30.1.103

Fitzpatrick, D. T., \& Shannon, S. (1992). Health-risk behaviors of Army aircrew. Journal of Occupational Medicine, 34, 810-814. Retrieved from http://journals.lww.com

Forgas, L. B., Meyer, D. M., \& Cohen, M. E. (1996). Tobacco use habits of naval personnel during Desert Storm. Military Medicine, 161, 165-168. Retrieved from http://www.ncbi.nlm.nih.gov

Grasser, J. A., \& Childers, E. (1997). Prevalence of smokeless tobacco use and clinical oral leukoplakia in a military population. Military Medicine, 162, 401-404. Retrieved from http://www. ncbi.nlm.nih.gov

Haddock, C. K., Hoffman, K., Taylor, J. E., Schwab, L., Poston, W. S. C., \& Lando, H. A. (2008). An analysis of messages about tobacco in the Military Times magazine. Nicotine \& Tobacco Research, 10, 1191-1197. doi:10.1080/1462220080216 3126

Haddock, C. K., Taylor, J. E., Hoffman, K. M., Poston, W. S. C., Peterson, A., Lando, H. A., et al. (2009). Factors which influence tobacco use among junior enlisted personnel in the United States Army and Air Force: A formative research study. American Journal of Health Promotion, 23, 241-246. doi:10.4278/ajhp. 070919100

Haddock, C. K., Vander Weg, M., DeBon, M., Klesges, R. C., Talcott, G. W., Lando, H., et al. (2001). Evidence that smokeless tobacco use is a gateway for smoking initiation in young adult males. Prevention Medicine, 32, 262-267. doi:10.1006/pmed. 2000.0802

Hatsukami, D. K., \& Severson, H. H. (1999). Oral spit tobacco: Addiction, prevention and treatment. Nicotine \& Tobacco Research, 1, 21-44. doi:10.1080/14622299050011131

Hepburn, M. J., Johnson, J. M., Ward, J. A., \& Longfield, J. N. (2000). A survey of smoking cessation knowledge, training, and practice among U.S. Army general medical officers. American Journal of Preventive Medicine, 18, 300-304. doi:10.1016/S07493797(00)00123-9

Institute of Medicine. (2009). Combating tobacco use in military and veteran populations. Washington, DC: The National Academies Press.

International Agency for Research on Cancer. (2007). Smokeless tobacco and some tobacco-specific N-nitrosamines. In IARC monographs on the evaluation of carcinogenic risks to humans (Vol. 89; pp. 57-60). Lyons, France: IARC Working Group on the Evaluation of Carcinogenic Risks to Humans.

Joseph, A. M., Miggli, M., Pearson, K. C., \& Lando, H. (2005). The cigarette manufacturers' efforts to promote tobacco to the
U.S. military. Military Medicine, 170, 874-880. Retrieved from http://www.ingentaconnect.com

Kenny, K. K., Quigley, N. C., \& Regennitter, F. J. (1996). Survey of smokeless tobacco use in basic trainees and armor basic course officers. Military Medicine, 161, 37-42. Retrieved from http://www.ncbi.nlm.nih.gov

Klesges, R. C., DeBon, M., Vander Weg, M. W., Haddock, C. K., Lando, H. A., Relyea, G. E., et al. (2006). Efficacy of a tailored tobacco control program on long-term use in a population of U.S. military troops. Journal of Consulting and Clinical Psychology, 74, 295-306. doi:10.1037/0022-006X.74.2.295

Klesges, R. C., Ebbert, J. O., Morgan, G. D., Sherrill-Mittleman, D., Asfar, T., Talcott, W. G., et al. (2011). Impact of differing definitions of dual tobacco use: Implications for studying dual use and a call for operational definitions. Nicotine \& Tobacco Research, 13, 523-531. doi:10.1093/ntr/ntr032

Klesges, R. C., Sherrill-Mittleman, D., Ebbert, J. O., Talcott, G. W., \& DeBon, M. (2010). Tobacco use harm reduction, elimination, and escalation in a large military cohort. American Journal of Public Health, 100, 2487-2492. doi:10.2105/AJPH.2009.175091

Knapik, J. J., Reynolds, K., \& Barson, J. (1999). Risk factors for foot blisters during road marching: Tobacco use, ethnicity, foot type, previous illness, and other factors. Military Medicine, 164, 92-97. Retrieved from http://www.ncbi.nlm.nih.gov

Lando, H. A., Haddock, C. K., Klesges, R. C., Talcott, G. W., \& Jensen, J. (1999). Smokeless tobacco use in a population of young adults. Addictive Behaviors, 24, 431-437. doi:10.1016/ S0306-4603(98)00058-6

Martin, G. C., Brown, J. P., Eifler, C. W., \& Houston, G. D. (1999). Oral leukoplakia status six weeks after cessation of smokeless tobacco use. Journal of the American Dental Association, 130, 945-954. Retrieved from http://jada.ada.org

McClellan, S. F., Olde, B. A., Freeman, D. H., Mann, W. F., \& Rotruck, J. R. (2010). Smokeless tobacco use among military flight personnel: A survey of 543 aviators. Aviation, Space, and Environmental Medicine, 81, 575-580. doi:10.3357/ASEM.2317. 2010

Morgan, B. J. (2001). Evaluation of an educational intervention for military tobacco users. Military Medicine, 166, 1094-1098. Retrieved from http://www.ncbi.nlm.nih.gov

Peterson, A. L., Severson, H. H., Andrews, J. A., Gott, S. P., Cigrang, J. A., Gordon, J. S., et al. (2007). Smokeless tobacco use in military personnel. Military Medicine, 172, 1300-1305. Retrieved from http://www.ingentaconnect.com

Piano, M. R., Benowitz, N. L., FitzGerald, G. A., Corbridge, S., Heath, J., Hahn, E., et al. (2010). Impact of smokeless tobacco products on cardiovascular disease: Implications for policy, prevention, and treatment. A policy statement from the American Heart Association. Circulation, 122, 1520-1544. doi:10.1161/ CIR.0b013e3181f432c3

Rae Olmsted, K. L., Bray, R. M., Reyes Guzman, C. M., Williams, J., \& Kruger, H. (2011). Overlap in use of different 
types of tobacco among active duty military personnel. Nicotine \& Tobacco Research, 13, 691-698. doi:10.1093/ntr/ntr060

Severson, H. H., Peterson, A. L., Andrews, J. A., Gordon, J. S., Cigrang, J. A., Danaher, B. G., et al. (2009). Smokeless tobacco cessation in military personnel: A randomized controlled trial. Nicotine \& Tobacco Research, 11, 730-738. doi:10.1093/ntr/ntp057

Shipley, R., Tresch, K., Tracey, S., \& Wilcox, A. (2002). The Buckley tobacco cessation program: Helping smokers quit. Federal Practitioner, 13-22. Retrieved from http://www.fedprac.com

Sridhar, A., Deuster, P. A., Becker, W. J., Coll, R., O’Brien, K. K., \& Bathalon, G. (2003). Health assessment of U.S. Army rangers. Military Medicine, 168, 57-62. Retrieved from http://www. ncbi.nlm.nih.gov

Substance Abuse and Mental Health Services Administration. (2010). Results from the 2009 National Survey on Drug Use and Health: Volume I. Summary of National Findings. (Office of Applied Studies, NSDUH Series H-38A, HHS Publication No. SMA 10-4856Findings). Rockville, MD. Retrieved from http://www.oas.samhsa.gov

Tomar, S. L., Alpert, H. R., \& Connolly, G. N. (2010). Patterns of dual use of cigarettes and smokeless tobacco among US males: Findings from national surveys. Tobacco Control, 19, 104-109. doi:10.1136/tc.2009.031070

Trent, L. K., Hilton, S. M., \& Melcer, T. (2007). Premilitary tobacco use by male Marine Corps recruits. Military Medicine, 172,1077-1083. Retrieved fromhttp://www.ingentaconnect.com

U.S. Census Bureau. (2011). National security \& veteran affairs. In U.S. Census Bureau (Ed.), The 2011 statistical abstract. (section 10). Retrieved from http://www.census.gov/compendia/ statab/2011edition.html

U.S. Department of Defense. (2011). Armed forces strength figures for April 30, 2011. DoD Personnel and Procurement Statistics, Personnel, Service Totals. Retrieved fromhttp://siadapp.dmdc.osd.mil/

U.S. Department of Health and Human Services, National Institutes of Health, National Cancer Institute. (2010). Tobacco control research: Smokeless tobacco: Measures and determinants of smokeless tobacco use, prevention, and cessation: DCCPS grants awarded in response to an NIH RFA initiative. Retrieved from http://cancercontrol.cancer.gov/tcrb/
Vander Weg, M. W., DeBon, M., Peterson, A. L., SherrillMittleman, D., Klesges, R. C., \& Relyea, G. E. (2005). Prevalence and correlates of lifetime smokeless tobacco use in female military recruits. Nicotine \& Tobacco Research, 7, 431-441. doi:10.1080/14622200500125781

Vander Weg, M. W., Peterson, A. L., Ebbert, J. O., DeBon, M., Klesges, R. C., \& Haddock, C. K. (2008). Prevalence of alternative forms of tobacco use in a population of young adult military recruits. Addictive Behaviors, 33, 69-82. doi:10.1016/j.addbeh.2007.07.005

Walsh, M. M., Langer, T. J., Kavanagh, N., Mansell, C., MacDougal, W., Kavanagh, C., et al. (2010). Smokeless tobacco cessation cluster randomized trial with rural high school males: Intervention interaction with baseline smoking. Nicotine \& Tobacco Research, 12, 543-550. doi:10.1093/ntr/ntq022

Ward, K. D., Vander Weg, M. W., Klesges, R. C., Kovach, K. W., Elrod, M. C., DeBon, M., et al. (2003). Characteristics of highly physically active smokers in a population of young adult military recruits. Addictive Behaviors, 28, 1405-1418. doi:10.1016/ S0306-4603(02)00267-8

Warnakulasuriya, S. (2009). Global epidemiology of oral and oropharyngeal cancer. Oral Oncology, 45, 309-316. doi:10.1016/ j.oraloncology.2008.06.002

Warnakulasuriya, S., Dietrich, T., Bornstein, M. M., Peidró, E. C., Preshaw, P. M., Walter, C., et al. (2010). Oral health risks of tobacco use and effects of cessation. International Dental Journal, 60,7-30. doi:10.1922/IDJ_2532Warnakulasuriya24

Wetter, D. W., McClure, J. B., de Moor, C., Cofta-Gunn, L., Cummings, S., Cinciripini, P. M., et al. (2002). Concomitant use of cigarettes and smokeless tobacco: Prevalence, correlates, and correlates of tobacco cessation. Preventive Medicine, 34, 638-648. doi:10.1006/pmed.2002.1032

Williams, L., Hermesch, C., Gackstetter, G., Lando, H., \& Fiedler, E. (1996). Prevalence of tobacco use among first-term Air Force personnel before and after basic military training. Military Medicine, 161, 318-323. Retrieved from http://psycnet.apa.org

Yatsuya, H., \& Folsom, A. R. (2010). Risk of incident cardiovascular disease among users of smokeless tobacco in the atherosclerosis risk in communities (ARIC) study. American Journal of Epidemiology, 172, 600-605. doi:10.1093/aje/kwq191 